\title{
Saponinas esteroidales de la planta Agave brittoniana (Agavaceae) con actividad contra el parásito Trichomona vaginalis
}

\author{
José Orestes Guerra ${ }^{1}$, Alfredo Meneses ${ }^{2,5}$, Ana María Simonet ${ }^{3}$, Francisco Antonio Macías ${ }^{3}$, \\ Clara Nogueiras ${ }^{4}$, Alicia Gómez ${ }^{5} \&$ José A. Escario $^{5}$ \\ 1. Facultad de Química y Farmacia, Universidad Central de Las Villas, Cuba; jo@uclv.edu.cu \\ 2. Departamento de Parasitología, Centro de Bioactivos Químicos, Universidad Central de Las Villas, Cuba. \\ 3. Facultad de Ciencias, Universidad de Cádiz, España. \\ 4. Facultad de Química, Universidad de La Habana, Cuba. \\ 5. Departamento de Parasitología, Universidad Complutense de Madrid, España.
}

Recibido 24-X-2007. Corregido 16-VI-2008. Aceptado 16-VII-2008.

\begin{abstract}
Steroidal saponins from the plant Agave brittoniana with activity against the parasite Trichomona vaginalis. The genus Agave (Agavaceae), includes more than 300 species; around 16 of them show an homogeneous distribution throughout Cuba. Agave brittoniana (ssp. brachypus), is an endemic subspecies that grows in the central region of the country and its leaves are traditionally used in the treatment of parasitic diseases. The parasite Trichomonas vaginalis causes the disease known as trichomoniasis, that infects the genital tract. To test in vitro the plant against Trichomona vaginalis, the dried and powdered leaves were extracted three times with ethanol-water $(7: 3)$ by maceration at room temperature. The solvent was removed under reduced pressure and the extract was suspended in distilled water, defatted with $n$-hexane, and extracted with water-saturated $n$-butanol. After solvent removal, a portion of the $n$-butanol extract was hydrolyzed. After extraction with ethyl acetate the hydrolysis products were compared with authentic sapogenins samples using thin layer chromatography (TLC). Most of the sapogenins (yuccagenin and diosgenin) were isolated and their structures were confirmed. using nuclear magnetic resonance (NMR) experiments. The $n$-butanol extract was subjected to a separation process through column chromatography to obtain five fractions. After multiple separation processes by reversed phase high performance liquid chromatography (HPLC), the most active one produced one refined fraction that contained two saponins with the same aglycone (diosgenin) and one yuccagenin based saponin. Best results of the activity were obtained with the yuccagenin derived glycoside. Rev. Biol. Trop. 56 (4): $1645-$ 1652. Epub 2008 December 12.
\end{abstract}

Key words: Anti-parasitic, Agave brittoniana, Trichomona vaginalis, steroidal saponins.

El parásito Trichomonas vaginalis provoca la enfermedad conocida como trichomonosis o tricomoniasis, que es una infección de tracto genital y fue descubierta en el año 1826 por el investigador francés Donné (Michel 1995).

Esta enfermedad, que afecta a una gran cantidad de personas en todo el mundo, produce una vulvovaginitis de leve a grave que puede dañar la uretra y el endocervix (García.1993). Los parásitos no pueden vivir de forma natural sin estrecha relación con el tejido vaginal, poco después de llegar a éste proliferan y producen degeneración y descamación del epitelio vaginal, con infiltración leucocitaria y aumento de las secreciones (Llop et. al. 2001).

En el hombre, el microorganismo habita en la uretra, próstata y vesículas seminales, pero rara vez produce uretritis o algún otro síntoma (Krieger 1995), por lo tanto, las mujeres con frecuencia son contagiadas repetidamente por sus compañeros de relaciones sexuales ya que no saben que ellos están infectados. Los tratamientos más utilizados para combatir esta enfermedad están basados en el uso 
del Metronidazol, así como otros derivados de 5-nitroimidazoles (Inceboz et. al. 2004, Cudmore et. al.2004, Crowell et. al. 2003).

Sin embargo, el número de personas resistentes a estos medicamentos ha aumentado, lo cual indica que el parásito se está adaptando a los mismos por lo que se trabaja intensamente en la búsqueda de otros compuestos que sean eficaces. (Kulda et. al. 1982, Kulda et. al.1993, Kulda 1999, Meri et. al.. 2000)

En este sentido una alternativa es el uso de plantas medicinales por lo que en este trabajo se presentan los resultados de la evaluación de extractos y productos obtenidos de hojas de la planta endémica cubana Agave brittoniana Trel. subespecie brachypus (fam. Agavaceae) frente a este parásito.

\section{MATERIALES Y MÉTODOS}

Obtención del "crudo de saponinas": El material vegetal (hojas) fue colectado en las inmediaciones de la zona protegida "Cubanacán", en las afueras de la ciudad de Santa Clara. Este se cortó en pequeños pedazos que se secaron en una estufa a $40{ }^{\circ} \mathrm{C}$ hasta obtener un peso constante (aproximadamente 10 días) y se molió hasta polvo fino. Este polvo se maceró en una mezcla de etanol y agua (7:3 $\mathrm{v} / \mathrm{v}, 3 \mathrm{x} 48$ horas) a temperatura ambiente y en la oscuridad. Los extractos etanólicos se unieron y evaporaron a vacío.

Se disolvieron en agua $10 \mathrm{~g}$ del extracto etanólico y se extrajo varias veces en un embudo de decantación, primero con n-hexano (desengrase) y después con n-butanol saturado en agua, evaporando al vacío el disolvente (crudo de saponinas).

Hidrólisis del "crudo de saponinas": Se disolvieron 5,0 $\mathrm{g}$ del extracto de saponinas en $75 \mathrm{~mL}$ de etanol y se añadieron $75 \mathrm{~mL}$ de $\mathrm{HCl}$ de concentración molar equivalente $2 \mathrm{~mol} / \mathrm{L}$. La solución se reflujó durante tres horas después de lo cual se concentró hasta la tercera parte de su volumen inicial, se disolvió con igual cantidad de agua y se extrajo con acetato de etilo. La solución orgánica se secó y se concentró a sequedad.
Separación y caracterización de los aglicones: La separación se realizó utilizando la técnica de cromatografía de columna (CC) con silicagel como fase estacionaria. Como disolventes para la elusión se utilizaron n-hexano y mezclas de n-hexano/ acetato de etilo en proporciones $1: 1,3: 2$ y $2: 3$

Para la caracterización de los aglicones se utilizó la cromatografía de capa delgada (CCD), espectroscopia infrarroja (IR) y resonancia magnética nuclear protónica y de carbono $\left(\mathrm{RMN}{ }^{1} \mathrm{H}\right.$ y $\left.{ }^{13} \mathrm{C}\right)$.

Fraccionamiento del "crudo de saponinas": En una columna se cromatografiaron 10 $\mathrm{g}$ del crudo de saponinas utilizando silicagel fase inversa y como eluyentes mezclas de metanol: agua en proporciones 2:3, 3:2, 4:1 (v:v) y agua sucesivamente.

A partir de las fracciones de interés así obtenidas y mediante la utilización combinada de las técnicas cromatográficas de media (MPLC) y alta (HPLC) presión utilizando columnas de silicagel fase inversa y una mezcla MeOH: agua (8:2) como fase móvil, se obtuvieron los glicósidos que fueron evaluados.

Determinación de la actividad contra Trichomonas vaginalis: En un frasco estéril, se añadieron $90 \mathrm{~mL}$ de medio de cultivo "Diamond", $10 \mathrm{~mL}$ de suero fetal bovino, 500 UI de Penicilina y $50 \mathrm{mg}$ de Estreptomicina. Posteriormente se adicionó un inóculo de 2000 T. vaginalis $/ \mathrm{ml}$ y se incubó a $37^{\circ} \mathrm{C}$ durante 48 horas.

A partir del cultivo en masa de $48 \mathrm{~h}$ de incubación (en fase de crecimiento logarítmico), se preparó un cultivo madre ajustándose el mimo a una concentración de $1 \times 10^{5} \mathrm{~T}$. vaginalis $/ \mathrm{ml}$. El volumen de cultivo madre a preparar se calculó teniendo en cuenta la cantidad de tratamientos, grupo control, número de réplicas, volumen de cada réplica, volumen final de cultivo a preparar y la concentración inicial y final de $T$. vaginalis por $\mathrm{ml}$. Del cultivo madre se pasaron $2 \mathrm{ml}$ a tubos de cultivo de 13 x 100 y se incubaron a $37^{\circ} \mathrm{C}$ y $5 \%$ de $\mathrm{CO}_{2}$. Después de 6 horas de incubación, se realizó 
una valoración del crecimiento mediante conteo de células vivas en cámara de Neubauer a 6 tubos controles. De ser el crecimiento superior o cercano a $10^{5}$ células por $\mathrm{ml}$ se procede a añadir los productos a ensayar disueltos en agua a distintas concentraciones (tres réplicas de cada uno)

De la misma forma se procedió con una solución de metronidazol $(2,1$ y $0.5 \mu \mathrm{g} / \mathrm{ml})$ que se utilizó como control.

Para la determinación de los porcentajes de actividad citostática y de reducción se utilizaron los siguientes fórmulas:

ICC $=$ Media conteo (24 - 48 horas $) /$ media conteo del control 6 horas

$\mathrm{ICP}=$ Media conteo producto / media conteo del control 6 horas

$\%$ de Crecimiento producto $=$ ICP $.100 /$ ICC

$\%$ de Actividad Citostática $=$ 100 - \% de crecimiento producto (cuando se cumple ICP $>=1$ )

$\%$ de reducción $=100-\%$ de crecimiento producto (cuando se cumple ICP $<1$ )

ICC $=$ Índice de crecimiento del control

$\mathrm{ICP}=$ Índice de crecimiento del producto

$\%$ de reducción $=\%$ de actividad citocida

Cuando el índice de crecimiento de cada producto es menor a 1 , se calcula $\%$ de reducción, por el contrario si mayor o igual a 1 se procede a calcular el \% de actividad citostática.

A los productos analizados se les determinó su actividad citocida (\% de reducción), cuando el cultivo tiene menos parásitos que el inóculo inicial, o su actividad citostática ( $\%$ de actividad citostática), en el caso contrario. El primero de estos parámetros es el que corresponde a los mejores resultados.

\section{RESULTADOS}

Estudio del extracto n-butanólico: A partir de $10 \mathrm{~g}$ de extracto hidroalcohólico se obtuvieron $6.2 \mathrm{~g}$ del crudo de saponinas que resultó ser activo frente a Trichomona vaginalis. Como resultado de la hidrólisis y el estudio cromatográfico se constató la presencia de Clorogenina (5 $\alpha, 25 \mathrm{R}$ - espirostano-3 $\beta, 6 \alpha$-diol), Hecogenina (3 $\beta$-hidroxi-5 $\alpha$, 25R-espirostan-

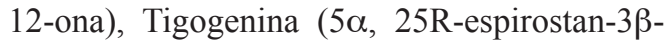
ol), Rockogenina (5 $\alpha, 25 \mathrm{R}$-espirostano-3 $\beta$, $12 \beta$-diol), $\beta$-Sitosterol ( $\Delta^{5}$-estigmastan-3 $\beta$ ol ), Diosgenina (25R-espirost-5-en-3 $\beta$-ol) y Yucagenina (25R-espirost-5-en-2 $\alpha, 3 \beta$-diol.). Los dos últimos, que resultaron ser los más abundantes, fueron separados por $\mathrm{CC}$ y se registraron sus espectros IR y RMN para confirmar su estructura,

Estudio de las fracciones obtenidas del crudo de saponinas: En el proceso de separación de este extracto se obtuvieron varias fracciones, tres de ellas (F-II, F-III y F-IV) constituidas por glicósidos.

Estas fueron evaluadas frentea Trichomonas vaginalis a concentraciones de 500, 100 y 10 $\mu \mathrm{g} / \mathrm{ml}$ (Cuadro 1; Cuadro 2).

A partir de esto preparamos un nuevo experimento con F-III y F-IV que son las fracciones que mostraron los mejores resultados. En este caso se utilizaron concentraciones de $40,20,10$ y $5 \mu \mathrm{g} / \mathrm{ml}$, para aproximarnos al valor de la mínima concentración letal.

Como apreciamos en el Cuadro 3, a menores concentraciones es F-IV quien mantiene su efectividad por lo que se continuó el trabajo con ésta aislando los glicósidos mayoritarios que ya a habían sido anteriormente caracterizados (Macías et. al.) y que responden a los nombres de Agabrittonósidos A, B y C que son saponinas esteroidales derivadas de la Yucagenina, la primera y de la Diosgenina, las dos restantes (Fig. 1). 
CUADRO 1

Datos de los espectros de $R M N H^{l}$ y $C^{13}$ de la yucagenina y diosgenina en $\mathrm{CDCl}_{3}$

TABLE 1

Data from the spectra $\mathrm{H}^{1} \mathrm{NMR}$ and $\mathrm{C}^{13}$ of yuccagenin and diosgenin in $\mathrm{CDCl}_{3}$

$\begin{array}{cccccc}\text { Posición } & \text { Yucagenina } \delta_{\mathrm{C}} & \text { Diosgenina } \delta_{\mathrm{C}} & \text { Posición } & \text { Yucagenina } \delta_{\mathrm{C}} & \text { Diosgenina } \delta_{\mathrm{C}} \\ 1 & 39,1 & 37,2 & 15 & 31,8 & 32,0 \\ 2 & 72,3 & 31,5 & 16 & 80,9 & 80,8 \\ 3 & 76,6 & 71,6 & 17 & 62,0 & 62,1 \\ 4 & 44,9 & 44,2 & 18 & 16,3 & 16,3 \\ 5 & 139,5 & 140,8 & 19 & 20,4 & 19,4 \\ 6 & 122,0 & 121,3 & 20 & 41,6 & 41,4 \\ 7 & 32,0 & 32,0 & 21 & 14,5 & 14,5 \\ 8 & 30,8 & 31,4 & 22 & 109,5 & 31,4 \\ 9 & 49,9 & 50,0 & 23 & 31,3 & 28,8 \\ 10 & 38,3 & 37,2 & 24 & 28,8 & 30,2 \\ 11 & 20,9 & 20,8 & 25 & 30,3 & 66,8 \\ 12 & 39,7 & 39,8 & 26 & 66,9 & 17,1\end{array}$

${ }^{1} \mathbf{H} \mathbf{R M N}\left(\mathrm{CDCl}_{3}\right){ }^{13} \mathbf{C} \mathbf{R M N}\left(\mathrm{CDCl}_{3}\right)$

Yucagenina: $\delta 3,59(1 \mathrm{H}, \mathrm{H}-2) ; 3,32(1 \mathrm{H}, \mathrm{H}-3) ; 5,38(1 \mathrm{H}, d, \mathrm{~J}=5,2 \mathrm{~Hz}, \mathrm{H}-6) ; 4,41(1 \mathrm{H}, d d d, \mathrm{H}-16), 3,37(1 \mathrm{H}, d d \mathrm{~J}=10,6$; $11,0 \mathrm{~Hz}, \mathrm{H}-26) ; 3,48(1 \mathrm{H}, d d \mathrm{H}-26) ; 1,12(3 \mathrm{H}, d, \mathrm{~J}=6,8 \mathrm{~Hz}, \mathrm{H}-21) ; 0,67$ (3H, $d, \mathrm{~J}=5,9 \mathrm{~Hz}, \mathrm{H}-27) ; 0,82(3 \mathrm{H}, s, \mathrm{H}-18) ; 1,08$ (3H, $s, \mathrm{H}-19)$.

Diosgenina: $\delta 5,37(1 \mathrm{H}, d, \mathrm{~J}=5,3 \mathrm{~Hz}, \mathrm{H}-6) ; 4,43(1 \mathrm{H}, d d d, \mathrm{H}-16), 3,38(1 \mathrm{H}, d d \mathrm{~J}=10,6 ; 11,0 \mathrm{~Hz}, \mathrm{H}-26) ; 3,48$ (1H, $m \mathrm{H}-26)$; $1,12(3 \mathrm{H}, d, \mathrm{~J}=6,8 \mathrm{~Hz}, \mathrm{H}-21) ; 0,67(3 \mathrm{H}, d, \mathrm{~J}=5,9 \mathrm{~Hz}, \mathrm{H}-27) ; 0,84(3 \mathrm{H}, s, \mathrm{H}-18) ; 1,02$ (3H, $s, \mathrm{H}-19)$.
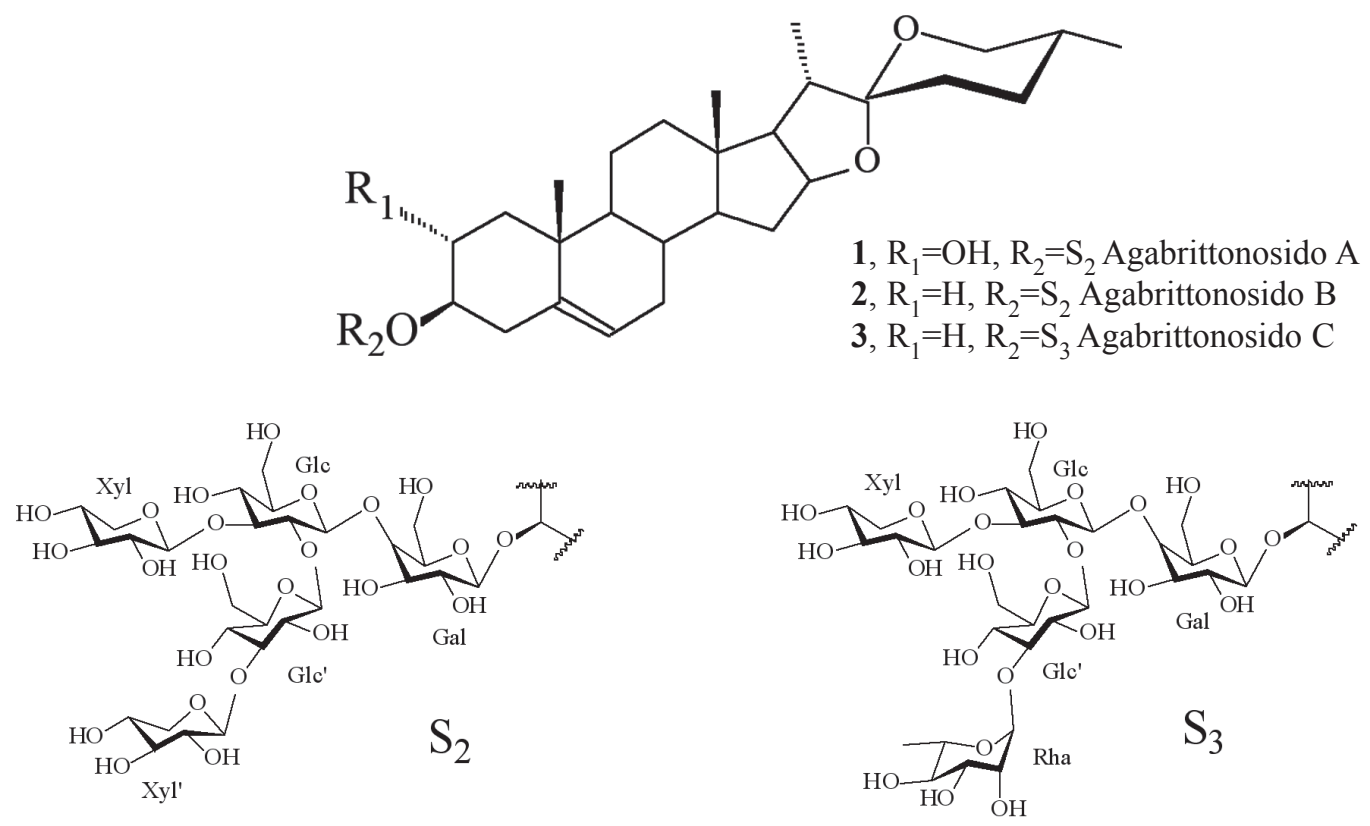

Fig. 1. Estructuras de las saponinas aisladas / Fig. 1. Structure of the isolated saponins. 
CUADRO 2

Resultados de la evaluación "in vitro" frente a Trichomonas vaginalis a concentraciones de 500, 100 y $10 \mu \mathrm{g} / \mathrm{ml}$

TABLE 2

Results of the "in vitro" assessment against Trichomonas vaginalis at 500, 100 and $10 \mu \mathrm{g} / \mathrm{ml}$ concentrations

\begin{tabular}{|c|c|c|c|c|c|}
\hline \multirow[t]{2}{*}{ Fracción } & \multirow{2}{*}{$\begin{array}{c}\text { Concent. } \\
\mu \mathrm{g} / \mathrm{ml}\end{array}$} & \multicolumn{2}{|c|}{24 Horas } & \multicolumn{2}{|c|}{48 Horas } \\
\hline & & $\%$ Reduc. & $\%$ Act. Citost. & $\%$ Reduc. & $\%$ Act. Citost. \\
\hline \multirow[t]{3}{*}{ F-II } & 500 & & 10.82 & & 63.45 \\
\hline & 100 & & 0.00 & & \\
\hline & 10 & & 0.00 & & \\
\hline \multirow[t]{3}{*}{ F-III } & 500 & 100.00 & & 100.00 & \\
\hline & 100 & 100.00 & & 100.00 & \\
\hline & 10 & & 0.00 & & 0.00 \\
\hline \multirow[t]{3}{*}{ F-IV } & 500 & 100.00 & & 100.00 & \\
\hline & 100 & 100.00 & & 100.00 & \\
\hline & 10 & 99.69 & & 99.68 & \\
\hline \multirow[t]{3}{*}{ Metronidazol } & 2 & 100 & & 100.00 & \\
\hline & 1 & 99.42 & & 99.76 & \\
\hline & 0.5 & 98.65 & & 98.04 & \\
\hline
\end{tabular}

CUADRO 3

Resultados de la evaluación "in vitro" de las fracciones III y IV a concentraciones de 40, 20, 10 y $5 \mu \mathrm{g} / \mathrm{ml}$

TABLE 3

Results of the "in vitro" assessment of the III and IV fractions at 40, 20, 10 and $5 \mu \mathrm{g} / \mathrm{ml}$ concentrations

\begin{tabular}{|c|c|c|c|c|c|}
\hline \multirow[t]{2}{*}{ Fracción } & \multirow{2}{*}{$\begin{array}{c}\text { Concent. } \\
\mu \mathrm{g} / \mathrm{ml}\end{array}$} & \multicolumn{2}{|c|}{24 Horas } & \multicolumn{2}{|c|}{48 Horas } \\
\hline & & $\%$ Reduc. & $\%$ Act. Citost. & \% Reduc. & $\%$ Act. Citost. \\
\hline \multirow[t]{4}{*}{ F-III } & 40 & 98.75 & & 95,07 & \\
\hline & 20 & & 2.62 & & 18.21 \\
\hline & 10 & & 0.00 & & 17.22 \\
\hline & 5 & & 0.00 & & 0.00 \\
\hline \multirow[t]{4}{*}{ F-IV } & 40 & 100.00 & & 100,00 & \\
\hline & 20 & 100.00 & & 100,00 & \\
\hline & 10 & 99.74 & & 98,84 & \\
\hline & 5 & & 71.28 & & 21.52 \\
\hline
\end{tabular}

Realizamos un nuevo experimento utilizando el producto Agabrittonósido A y la mezcla de Agabrittonósidos $\mathrm{B}$ y $\mathrm{C}$, a concentraciones de 100, 10 y $1 \mu \mathrm{g} / \mathrm{ml}$ (Cuadro 4).

De los glicósidos probados el Agabrittonósido A presentó una elevada actividad a concentraciones de 100 y $10 \mu \mathrm{g} / \mathrm{ml}$ con porcentajes de reducción de 100 o muy cercanos, indicador que disminuyó al trabajar a concentraciones de $1 \mu \mathrm{g} / \mathrm{ml}$ lo que constituye una actividad moderada si la comparamos patrón de metronidazol, que mantiene altos valores de reducción incluso a $0.5 \mu \mathrm{g} / \mathrm{ml}$ (Cuadro 5).

\section{DISCUSIÓN}

Estudio del extracto n-butanólico: Teniendo en cuenta que el extracto n-butanólico presentó actividad frente a Trichomona vaginalis se procedió a la caracterización del mismo. De acuerdo a la procedencia de este 
CUADRO 4

Resultados de la evaluación "in vitro" de los glicósidos mayoritarios en la fracción IV

TABLE 4

Results of the "in vitro" assessment of the principal glycosides in the IV fraction

\begin{tabular}{|c|c|c|c|c|c|}
\hline \multirow[t]{2}{*}{ Productos } & \multirow{2}{*}{$\begin{array}{c}\text { Concent. } \\
\mu \mathrm{g} / \mathrm{ml}\end{array}$} & \multicolumn{2}{|c|}{24 Horas } & \multicolumn{2}{|c|}{48 Horas } \\
\hline & & $\%$ Reduc. & $\%$ Act. Citost. & $\%$ Reduc. & $\%$ Act. Citost. \\
\hline \multirow[t]{3}{*}{ Agabrittonósido A } & 100 & 100.00 & & 100.00 & \\
\hline & 10 & 99.71 & & 97.31 & \\
\hline & 1 & & 21.21 & & 13.59 \\
\hline \multirow[t]{3}{*}{ Mezcla de Agabrittonósidos B y C } & 100 & 100.00 & & 100.00 & \\
\hline & 10 & & 81.34 & & 59.21 \\
\hline & 1 & & 4.94 & & 10.95 \\
\hline
\end{tabular}

CUADRO 5

Resultados de la acción del metronidazol frente a Trichomonas vaginalis

TABLE 5

Results of the metronidazol action against Trichomonas vaginalis

\begin{tabular}{|c|c|c|c|c|c|}
\hline \multirow[t]{2}{*}{ Producto } & \multirow{2}{*}{$\begin{array}{c}\text { Concent. } \\
\mu \mathrm{g} / \mathrm{ml}\end{array}$} & \multicolumn{2}{|c|}{24 Horas } & \multicolumn{2}{|c|}{48 Horas } \\
\hline & & $\%$ Reduc. & $\%$ Act. Citost & $\%$ Reduc. & $\%$ Act. Citost \\
\hline Metronidazol & 2 & 99.67 & & 100.00 & \\
\hline & 1 & 99.18 & & 99.72 & \\
\hline & 0.5 & 98.19 & & 98.79 & \\
\hline
\end{tabular}

producto así como los resultados de la determinación cuantitativa de los metabolitos (Guerra et. al.) se constató un alto contenido de saponinas esteroidales.

De este extracto fueron aisladas las agliconas mayoritarias, confirmándose su naturaleza a partir del estudio espectroscópico.

En el espectro IR de la Diosgenina se apreciaron las bandas características de la vibración de valencia $\mathrm{O}-\mathrm{H}$ en $3400 \mathrm{~cm}^{-1}$ y C-O en $1050 \mathrm{~cm}^{-1}$. También se apreciaron las señales en $850,900,920$ y $985 \mathrm{~cm}^{-1}$, con mayor intensidad en la de $900 \mathrm{~cm}^{-1}$ respecto a la situada en $920 \mathrm{~cm}^{-1}$ lo cual confirma una configuración "R" alrededor del C- 25.

En el espectro protónico se observa como señal más desblindada un doblete en 5,37 ppm que asignamos al protón enlazado a C-6 $\left(\mathrm{sp}_{2}\right)$ que acopla con los dos protones de $\mathrm{C}-7$. $\mathrm{La}$ señal en 4,43 ppm corresponde a $\mathrm{H}-16 \alpha$ y es debido al acoplamiento con los protones $15 \beta$, $15 \alpha$ y $17 \beta$. Las señales que aparecen en 3,48 y 3,38 ppm se asignaron a H-26 $\beta$ y H-26 $\alpha$ respectivamente.

Las señales de los protones correspondientes a los metilos C-18 y C-19 que aparecen como singletes y la de los protones correspondientes a los metilos C-21 y C-27 que aparecen como dobletes, se corresponden a las reportadas en la literatura para la Diosgenina.

En el espectro de $\mathrm{RMN}{ }^{13} \mathrm{C}$ se destacan como la señales más desblindadas las situadas en 140,8 y 121,3 que asignamos a C-5 y C-6 respectivamente, lo cual se corresponde con el hecho que estos carbonos poseen hibridación $\mathrm{sp}_{2}$.

En ese orden le sigue una señal en 109,3 ppm que corresponde a $\mathrm{C}-22$, que es el punto 
de unión entre los anillos $\mathrm{E}$ y $\mathrm{F}$ y está directamente unido a dos átomos de oxígeno. Las señales de los átomos de carbono del anillo $\mathrm{F}$; C-23, C-24, C-25 , C-26 y C-27 que son diagnósticas de estos compuestos aparecen en 31,4 ; 28,8; 30,2; 66,8 y 17,1 ppm respectivamente indicándonos que estamos en presencia de un (25R) espirostano.

En el caso de la Yucagenina el espectro IR mostró las bandas características de la vibración de valencia $\mathrm{O}-\mathrm{H}$ en $3400 \mathrm{~cm}^{-1}$ y C-O en $1050 \mathrm{~cm}^{-1}$. También se apreciaron las señales en $850,900,920$ y $985 \mathrm{~cm}^{-1}$, con mayor intensidad en la de $900 \mathrm{~cm}^{-1}$ respecto a la situada en $920 \mathrm{~cm}^{-1}$ lo cual confirma una configuración "R" alrededor del C- 25. Este espectro es perfectamente superponible con el del patrón.

En el espectro de RMN protónico se observa como la señal más desblindada un dupleto en 5,38 que asignamos al protón enlazado a C-6 ( $\left.\mathrm{sp}_{2}\right)$ que acopla con los dos $\mathrm{H}$ del C-7. El cuarteto que aparece en 4,41 ppm corresponde a H-16 y es debido al acoplamiento con los protones $15 \beta, 15 \alpha$ y $17 \beta$. Las señales que aparecen en 3,59 y $3,32 \mathrm{ppm}$ la asignamos a los protones $\mathrm{H}-2$ y $\mathrm{H}-3$ respectivamente, en 3,48 ppm aparece un doblete de doblete correspondiente a H-26ß y otra señal en 3,37 ppm asignada a $\mathrm{H}-26 \alpha$. Las señales de los protones correspondientes a los metilos C-18 y C-19 que aparecen como singletes y la de los protones correspondientes a los metilos C-21 y C-27 que aparecen como dobletes se corresponden a las reportadas en la literatura para la Yucagenina.

En el espectro de RMN ${ }^{13} \mathrm{C}$ de este compuesto se destacan como las señales más desblindadas las situadas en 139,5 y 122,0 ppm que asignamos a C-5 y C-6 respectivamente lo cual se corresponde con el hecho que estos carbonos poseen hibridación $\mathrm{sp}_{2}$.

En ese orden le sigue una señal en 109,5 ppm que corresponde a C-22, que es el punto de unión entre los anillos $\mathrm{E}$ y $\mathrm{F}$ y está directamente unido a dos átomos de oxígeno. Las señales de los átomos de carbono del anillo F; C-23, C-24, C-25 , C-26 y C-27 que son diagnósticas de estos compuestos aparecen en 31,$3 ; 28,8 ; 30,3 ; 66,9$ y 17,1 ppm respectivamente indicándonos que estamos en presencia de un (25R) espirostano.

De esta forma se confirmó la estructura de los aglicones que en mayor proporción aparecen en el extracto lo que hizo suponer que también la mayoría de los glicósidos procedieran de estas.

En el proceso de fraccionamiento inicialmente fueron obtenidas cinco fracciones, la primera, un sólido de color pardo claro formado por los compuestos más polares del extracto, probablemente azúcares libres, mientras que la última, un sólido grasiento de color verde oscuro formada por clorofilas y otros constituyentes menos polares. Las más activas fueron las fracciones intermedias (F-II, F-III y F-IV), constituidas por los glicósidos.

Estudio de las fracciones obtenidas del crudo de saponinas: La fracción F-IV es la que mejor actividad mostró en este experimento, pues aún a una concentración de 10 $\mu \mathrm{g} / \mathrm{ml}$ mantuvo un elevadísimo porcentaje de reducción, mientras que en F-III la actividad cae bruscamente de $100 \%$ de reducción a 100 $\mu \mathrm{g} / \mathrm{ml}$ hasta ser nula a $10 \mu \mathrm{g} / \mathrm{ml}$. La Fracción II fue la de peor comportamiento pues a la máxima concentración tuvo actividades citostáticas de sólo 10.82 y $63.45 \%$ a las 24 y 48 horas respectivamente y su actividad fue nula a concentraciones inferiores. De acuerdo a su comportamiento cromatográfico, la polaridad de los glicósidos disminuye de la fracción II a la IV, lo que indica que la actividad fue disminuyendo en la medida que aumentó la polaridad de las saponinas.

Estudio de los productos obtenidos: De la fracción IV pudo aislarse el Agabrittonósido A y una mezcla de los compuestos Agabrittonósido B y C. El primero es una saponina derivada de la yucagenina (Fig. 1) y presentó una elevada actividad a concentraciones de 100 y 10 $\mu \mathrm{g} / \mathrm{ml}$ con porcentajes de reducción de 100 o muy cercanos, indicador que disminuyó al trabajar a concentraciones de $1 \mu \mathrm{g} / \mathrm{ml}$ lo que constituye una actividad moderada si la comparamos patrón de metronidazol, que mantiene 
altos valores de reducción incluso a $0.5 \mu \mathrm{g} /$ $\mathrm{ml}$ (Cuadro 5). Los glicósidos que componen la mezcla presenta como aglicón común la diosgenina y tuvieron un peor comportamiento, pues la elevada actividad que presentan a $100 \mu \mathrm{g} / \mathrm{ml}$ cae rápidamente al disminuir la concentración.

Es de destacar que estas diferencias en la actividad biológica son provocadas por cambios muy a pequeños en las estructuras de estos compuestos; el hidroxilo que está en la posición 2 del Agabrittonósido A no aparece en los otros glicósidos mientras que las cadenas glicosídicas sólo se diferencian en la sustitución de una xilosa terminal por rhamnosa para el Agabrittonósido C.

\section{RESUMEN}

El género Agave, familia Agavaceae, tiene más de 300 especies, con aproximadamente 16 distribuidas en toda Cuba. Una de ellas, el Agave brittoniana Trel. (ssp. brachypus), es una subespecie endémica y sus hojas son tradicionalmente utilizadas en el tratamiento de enfermedades parasitarias. Se realizaron estudios "in vitro" de la actividad de productos de esta planta frente a Trichomona vaginalis. Las hojas secas y pulverizadas fueron extraídas tres veces con una mezcla de etanol-agua (7: 3) mediante maceración a temperatura ambiente. El disolvente fue evaporado a presión reducida y el extracto fue suspendido en agua destilada, desengrasado con n-hexano, y extraído con n-butanol saturado con agua. Luego de una extracción con acetato de etilo, los productos de la hidrólisis fueron comparados con patrones de sapogeninas mediante la cromatografía de capa fina (CCD). Aislamos las sapogeninas mayoritarias (yuccagenina y diosgenina) y confirmamos sus estructuras utilizando técnicas de resonancia magnética nuclear. Por otra parte, el extracto n-butanólico fue sometido a un proceso de separación biodirigido mediante cromatografía de columna, obteniéndose cinco fracciones. Después de múltiples separaciones, la más activa rindió una fracción purificada con dos sapogeninas con el mismo aglicón (diosgenina) y un glicósido de yucagenina. Los mejores resultados de esta actividad fueron obtenidos con el glicósido derivado de la yucagenina

Palabras clave: Antiparasitario, Agave brittoniana, Trichomona vaginalis, sapogeninas esteroidales.

\section{REFERENCIAS}

Crowell, A. L., Sanders-Lewis, K. A. \& Secor, W. E. 2003. In vitro metronidazole and tricabendazole activities against metronidazole-resistant strains of Trichomonas vaginalis. Antimicrob. Agents Chemother. 47: 1407-1409.

Cudmore: S.: Delgaty: K. L.: Hayward-McClelland: Shannon: F.: Petrin: D. P. \& Garber: G. E. 2004. Treatmen of infections caused by metronidazoleresistant Trichomonas vaginalis. J. Chemother. 17: 783-793.

García: S. 1993. Diagnostic Medical Parasitology. American Society for Microbiology. Washington D.C. p. 84-91.

Guerra: J.O.: Nogueira: C.: Delgado: R. \& Hernández: O. 2001. Determinación cuantitativa de saponinas y azúcares reductores del Agave brittoniana T. Rev. Cub. Quím. 3: 37-42.

Inceboz: T.: Inceboz: U. \& Ozturk: S. 2004. Comparative in vitro cytotoxic effects of ornidazole: metronidazole and ciprofloxacin against Trichomonas vaginalis trophozoites. J. Chemother. 16: 459-462.

Krieger: J. N. 1995. Trichomoniasis in men: old issues and new data. Sex Transm. 22: 83-96.

Kulda: J. 1999. Trichomonads: hidrogenosomes and drug resistance. Int. J. Parasitol. 29. 199-212

Kulda: J.: Tachezy: J. \& Cerkasovova: A. 1993. In vitro induced anaerobic resistance to metronidazole in Trichomonas vaginalis. J Euk. Microbiol. 40: 262-9.

Kulda: J.: Vojtechowská: M.: Tachezy: J.: Demed: P. \& Kunzová E. 1982. Metronidazole resistance of Trichomonas vaginalis as a cause of treatment failure in trichomoniasis. Br J Vener Dis. 58: 394-399.

Llop Hernández: A.: Valdés-Dapena Vivanco: M. \& Zuazo Silva: J. L. 2001. Microbiología y Parasitología Médicas. Ed. Ciencias Médicas. La Habana p. 40.

Macías: F. A.: Guerra: J. O.: Simonet: A. M. \& Nogueira: C. 2007. Characterization of the fraction components using 1D TOCSY and 1D ROESY experiments. Four new spirostane saponins from Agave brittoniana Trel. spp. Brachypus. Magn. Reson. Chem. 45: 615-620

Meri: T.: Jokiranta: T. S.: Suponen: L. \& Meri: S. 2000. Resistance of Trichomonas vaginalis to metronidazole: Report of the first three cases from Finland and optimization of in vitro susceptibility testing under various oxygen concentrations. J.Clin.Microbiol. 38: 763-767.

Michel: R. 1995. Trichomoniasis. En Goldsmith, R.. \& Heyneman, D. (eds). Parasitología y Medicina Tropical. S.E., Bogotá. 